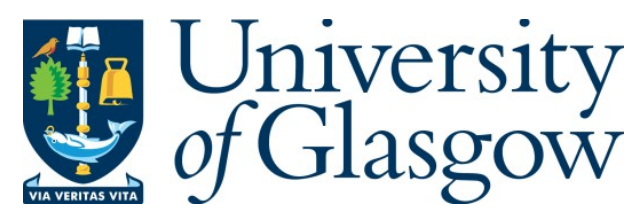

Sambo, Y., Shakir, M., Qaraqe, K., Serpedin, E., and Imran, M. (2014) Expanding cellular coverage via cell-edge deployment in heterogeneous networks: spectral efficiency and backhaul power consumption perspectives. IEEE Communications Magazine, 52(6), pp. 140-149.

There may be differences between this version and the published version. You are advised to consult the publisher's version if you wish to cite from it.

http://eprints.gla.ac.uk/136088/

Deposited on: 1 February 2017

Enlighten - Research publications by members of the University of Glasgow http://eprints.gla.ac.uk 


\title{
Expanding Cellular Coverage via Cell-Edge Deployment in Heterogeneous Networks: Spectral efficiency and backhaul power consumption perspectives
}

\author{
Yusuf A. Sambo ${ }^{1}$, Muhammad Z. Shakir ${ }^{2}$, Khalid A. Qaraqe ${ }^{2}$, \\ Erchin Serpedin ${ }^{3}$, Muhammad A. Imran ${ }^{1}$ \\ ${ }^{1}$ Center for Communication Systems Research (CCSR), University of Surrey, Guildford, UK \\ \{yusuf.sambo, m.imran\}@surrey.ac.uk \\ ${ }^{2}$ Electrical and Computer Engineering Dept., Texas A\&M University at Qatar, Doha \\ \{muhammad.shakir, khalid.qaraqe\} @qatar.tamu.edu \\ ${ }^{3}$ Electrical and Computer Engineering Dept., Texas A\&M University, College Station, TX \\ serpedindece.tamu.edu
}

\begin{abstract}
Heterogeneous small-cell networks (HetNets) are considered as a standard part of future mobile networks where operator/consumer deployed small-cells, such as femtocells, relays and distributed antennas (DAs), complement the existing macrocell infrastructure. This paper proposes the need oriented deployment of small-cells and device-to-device (D2D) communication around the edge of the macrocell such that the small-cell base stations (SBSs) and D2D communication serve the cell-edge mobile users, thereby expanding the network coverage and capacity. In this context, we present competitive network configurations namely: (i) femto-on-edge, (ii) DA-on-edge, (iii) relay-on-edge and (iv) D2Dcommunication-on-edge, where femto base stations, DA elements, relay base stations and D2D communication, respectively, are deployed around the edge of the macrocell. The proposed deployments ensure performance gains in the network in terms of spectral efficiency and power consumption by facilitating the cell-edge mobile users with small-cells and D2D communication. In order to calibrate the impact of power consumption on the system performance and network topology, this paper discusses the detailed breakdown of the end-to-end power consumption which includes backhaul, access and aggregation network power consumptions. Several comparative simulation results quantify the improvements in spectral efficiency and power consumption of the D2D-communication-on-edge configuration to establish a greener network over the other competitive configurations.
\end{abstract}

This publication was made possible by NPRP grant 4-1293-2-513 and NPRP grant 09-341-2-128 from the Qatar National Research Fund (a member of Qatar Foundation). The statements made herein are solely the responsibility of the authors. 


\section{Index Terms}

Heterogeneous small-cell networks (HetNets); cell-on-edge; femto-on-edge; relay-on-edge; distributed antenna-on-edge; D2D communication-on-edge; backhaul power consumption and spectral efficiency.

\section{INTRODUCTION}

There has been a paradigm shift in the design criteria of future networks, with the $5^{\text {th }}$ generation (5G) mobile system focusing on providing higher data rates, lower latency, energy efficiency (EE), increased number of simultaneous active mobile users and last but not the least, improved performance at cell-edges. However, the spectral efficiency (SE) of the cell-edge mobile user is often very poor and degrades the overall network capacity and coverage. This is due to higher path-loss effects at the cell-edge since the received signal power at the mobile user decays exponentially with distance from the associated base station (BS), usually located at the center of the macrocell. Different vital technologies like carrier aggregation, improved multiple-input multiple-output (MIMO) schemes (e.g., massive MIMO) and coordinated multipoint communication (CoMP), among others, have been explored to address these stringent requirements.

Small-cells, such as femtocells, relays, and distributed antennas (DAs), are deployed within a macrocell to improve the network coverage and SE of certain spots within the macrocell coverage. The resultant network is referred to as a heterogeneous small-cell network (HetNet) [1]. HetNets offer wireless coverage in environments with extensive diversity, ranging from outdoor to indoor environments such as office buildings, homes, and underground areas. Although the macrocell BS deployment requires cautious network planning, small-cell deployment does not essentially need more than geographic knowledge of the hotspots. Hence, the deployment of small-cells is relatively ad-hoc to serve areas with high traffic density and to improve coverage. HetNets enable flexible and low-cost deployments, thereby providing a uniform broadband experience to the mobile users anywhere within the network, especially the cell-edge mobile users.

A promising way of increasing the achievable rate in cellular communications is direct communication between closely located users, termed device-to-device (D2D) communication. Mobile devices involved in D2D communication form a direct link with each other without the need 
of routing data via the cellular access network, resulting in lower transmit power and end-toend delay, as well as freeing network resources. D2D communication also exhibits the gains of offloading traffic from the core network, usage of both uplink and downlink resources and extending the coverage area of cellular networks [2].

A typical HetNet with multiple small-cell technologies and D2D communication supporting the macrocell network architecture is shown in Fig. 1. The large cell provides ubiquitous coverage to the mobile users relatively closer to the macrocell BS while the small-cell network elements such as femtocells, relays, and DAs bring connectivity closer to the mobile users and thereby increasing the achievable capacity in the system. The D2D communication links, on the other hand, bring about a localized communication, where closely located mobile users are able to communicate and exchange data in an efficient and cost effective way.

This paper discusses the cell-edge deployment of small-cells and D2D communication around a macrocell such that the resultant configuration is referred to as cell-on-edge (COE) configuration for the SBSs and D2D-communication-on-edge (DCOE) for D2D communication, and also determines, based on simulation results, the preferable cell-edge deployment configuration in terms of the achievable SE, total power consumption and backhaul requirements.

\section{A. Cell-Edge Deployment: Some Benefits}

Limitations in cellular coverage, especially at the cell-edge, can be overcome by transmission over small-cell BSs (SBSs) which are positioned at the cell-edge or in a coverage hole, in order to compensate for the penetration losses caused by buildings, obstacles and sparsely populated areas over the distance. Similarly, D2D communication can be introduced at the cell-edge of macrocells to support the mobile users there. The COE and DCOE configurations have been shown to produce significant gains to the operators and mobile customers, including improved cell-edge coverage, increased network capacity to match cell-edge mobile user demands, enhanced end-user experience and reduced cost of delivering mobile broadband services to such cell-edge mobile users. In addition, cell-edge deployment also brings about reduced power consumption and interference in the network, as well as improved SE performance by shortening the transmitter- 
receiver distance. This reduces the transmit power of the mobile users as a result of a smaller path-loss, which in turn leads to a low interference regime.

\section{B. Paper Organization}

This paper is organized as follows: Section II presents the system model and network layout of the cell-edge deployments. The area spectral efficiency (ASE) and the backhaul power requirements for the four cell-edge configurations are quantified in Sections III and IV, respectively. The end-to-end power consumption of the HetNets, including the uplink and downlink power consumptions are presented in Section $\mathrm{V}$, in order to recommend the greener network configuration among the competitive cell-edge network configurations. Several simulation results are provided where deemed necessary, based on the simulation parameters summarized in Table I. Finally, conclusions are drawn in Section VI.

\section{System Model AND Network LAyOUT}

This section presents the system model and network layout of the four cell-edge deployments.

Consider a two-tier HetNet where small-cells are arranged or D2D communication is introduced around the cell-edge of the macrocell. The first tier of the HetNet consists of a macrocell BS with an omnidirectional antenna located at the center of the macrocell of radius $R_{m}$. The macrocell is assumed to have $H$ mobile users which are uniformly distributed between $R_{0}$ and $R_{m}$, such that $R_{0}$ is the minimum distance between a macrocell mobile user and its serving macrocell BS. The second tier of the HetNet comprises of $N$ low-power small-cells (e.g., femtocells, relays, and DAs) deployed around the edge of the macrocell to provide the coverage to the cell-edge mobile users such that each SBS has a coverage radius of $R_{s}$. The number of mobile users in each small-cell is given by $M=(H-L) / N$ where $L=\left(H\left(R_{1}^{2}-R_{0}^{2}\right)\right) / R_{m}^{2}$ and $R_{1}=R_{m}-R_{s}$. For the sake of comparison, the second tier of the network could also involve the introduction of D2D communication around the cell-edge of the macrocell. In these configurations, $L$ out of $H$ mobile users are uniformly distributed in the region between $R_{0}$ and $R_{1}$ and are exclusively served by the macrocell $\mathrm{BS}$. The remaining $H-L$ mobile users are 
served by the $N$ small-cells or employ D2D communication. A user distribution of 0.005 per $\mathrm{m}^{2}$ is assumed throughout the network, for all deployment scenarios. The number of small-cells per macrocell can be expressed from [3] as $N=4 R_{m} / R_{s}$.

In the following sub-sections, we introduce the small-cell technologies for cell-edge HetNet deployment.

\section{A. Femto-on-Edge (FOE)}

FOE is the HetNet deployment where femtocell BSs (FBSs) are arranged around the edge of the macrocell. Femtocells are small, low-power, subscriber/operator deployed independent BSs that provide a cost-effective way of offloading traffic from the macrocell network [4]. Femtocells facilitate the cell-edge users from the following perspectives:

- flexibility in terms of subscriber deployment and privacy;

- direct channel access with minimal delays and superior signal quality due to shorter distance;

- availability of cheap backhaul between the FBSs, installed by the subscribers in their local premises, and the mobile network operator, i.e., femtocells are usually connected directly to the core network via broadband backhaul links over the Internet e.g., by employing Selected IP Traffic Offload (SIPTO).

Femtocells have a typical coverage of less than $50 \mathrm{~m}$ and transmit power below $200 \mathrm{~mW}$. The short transmit-receive distance of femtocells results in a much lower transmit power requirement in both the uplink and downlink directions and a higher signal to interference plus noise ratio (SINR) [5]. HetNets with FOE deployment are expected to achieve substantial ASE gain compared with macrocell only networks [3].

\section{B. Distributed Antenna-on-Edge (DOE)}

DAs are deployed around the edge of the macrocell, in the DOE deployment. DAs are operator installed, spatially separated antenna elements that serve mobile users while remaining connected to the macrocell BS usually via dedicated high-speed fiber backhaul links. The DAs act as extensions of the macrocell $\mathrm{BS}$ and provide coverage to certain spots within the network. Each 
DA has a transmit power in the range $200 \mathrm{~mW}-2 \mathrm{~W}$ and has a relatively small coverage of up to a few hundred meters. DA nodes are geographically distributed across a network to reduce the access distance to macrocell BS and thereby reduce the effect of path-loss and hence, improve the SINR and coverage. Moreover, the DOE deployment has been shown to improve the cell-edge performance of networks [6]. Unlike femtocells, the DAs are connected to a primary macrocell BSs that serves as the processing unit and the DAs are highly unlikely to be installed by the mobile users. Such dedicated link establishes an additional backhaul between the DAs and macrocell BS [7].

\section{Relay-on-Edge (ROE)}

$\mathrm{ROE}$ is the HetNet configuration where relays are integrated with the macrocell network around the edge. Relays are relatively small, low-power BSs deployed for coverage expansion and to improve overall capacity of the network. Relays aid the mobile users by receiving and retransmitting their signals over the wireless backhaul between the relay BS (RBS) and the macrocell BS. However, the performance of relay transmissions is greatly affected by the associated delays in transmission, cascaded channel effects, collaborative strategies and relay selection processes [8]. Relays have a typical transmit power of $1 \mathrm{~W}-5 \mathrm{~W}$ and coverage of few hundred meters. Relay deployment is favorable in situations where a fiber infrastructure to the macrocell BS is non-existent and there is enough power supply available for the microwave backhaul network [9].

\section{D2D-Communication-on-Edge (DCOE)}

The DCOE deployment is a network configuration where D2D communication is introduced around the cell-edge of a macrocell. This deployment supports cell-edge users that have poor cellular coverage and SE. D2D communication in this paper is assumed to be enabled for users located within the region bounded by $R_{m} \pm R_{D 2 D}$ around the cell-edge, such that the maximum distance between any two randomly distributed D2D communication enabled mobile users is 20 $\mathrm{m}$ for $R_{D 2 D}=10 \mathrm{~m}$. This DCOE enabling range allows for realistic localized communication 
and efficient content distribution between mobile users within D2D communication proximity and higher SE due to lower path-loss. Although the macrocell BS exchanges control and synchronization signals with mobile users, there is a signaling overhead in D2D communication because the BS would have to measure the D2D communication link quality and determine whether or not it would be suitable to establish such device centric communication link [10]. However, this overhead is expected to be negligible compared to the gain of D2D communication if signaling over IP (SoIP) is enforced under such network.

\section{E. Carrier Deployment}

Carrier deployment in HetNets could be performed in one of two ways: co-channel deployment and dedicated carrier deployment. Co-channel deployment has the advantage of exploiting the full system bandwidth in both tiers of the HetNet but at the cost of interference between the tiers, i.e., cross-tier interference. Dedicated deployment, on the other hand, does not suffer from that as both tiers operate on separate bandwidths. However, this comes with the limitation of not being able to use the entire bandwidth in each tier of the HetNet, and the scheme may suffer from poor static/dedicated spectrum allocation. Therefore, the dedicated carrier deployment is more suitable in conditions where there is a large system bandwidth available (usually $\geq 20 \mathrm{MHz}$ ) and a high small-cell/D2D communication user density. In this paper, we assume a dedicated carrier deployment where spectrum partitioning is based on the ratio of the number of mobile users in each tier of the network. We also assume that each channel is allocated to a single mobile user in the macrocell, small-cells, and D2D communication region of the network.

\section{F. Interference Coordination}

In the dedicated carrier deployment, interference in the downlink of the macrocell is assumed to be from neighboring co-channel macrocell BSs transmitting on the same bandwidth. Similarly, in the downlink of the small-cells, interference is received from $N-1$ SBSs in the same deployment setting. This is because the small-cells reuse the same allocated bandwidth. However, in the uplink, interference is received at the macrocell BS from the mobile users in each of the 
neighboring co-channel macrocell and transmitting on the same channel. Similarly, in the uplink of the small-cells, interference is from the mobile users in the neighboring $N-1$ small-cells transmitting on the same channel. On the other hand, interference in the D2D communication region is assumed to be to/from the closest D2D communication user that is not participating in the desired D2D communication link.

\section{G. Power Control}

Power control (PC) is necessary to achieve a uniform SINR of mobile users at the reference BS in the uplink such that each mobile user is allowed to transmit with just enough power to neutralize the effect of path-loss between the mobile user and its serving BS. However, at a certain distance, the mobile user would have to transmit with maximum power to overcome the effect of path-loss. In this paper, mobile users served by the macrocell BS, femtocells, as well as those involved in D2D communication are assumed to employ the PC mechanism ${ }^{1}$. This is because the femtocell is a scaled-down version of a complete BS making it capable of controlling the mobile users associated with it to adapt their transmit powers, and the macrocell BS advices the D2D communication users on their transmit powers based on their proximity by sending transmit power control (TPC) signals. However, there is no form of PC in the DOE and the ROE deployments as relays and DAs are not capable of sending the TPC commands to the mobile users. Hence, the mobile users in the DOE and the ROE strategies are expected to transmit with the maximum power. We assume the 3GPP path-loss dependent models in the calculation of the mean received signal power at each mobile user in the downlink [2], while the two-slope path-loss model was used to compute the mean received signal power in the uplink transmissions, both at the macrocell BS and the SBS [3].

\section{AREA Spectral EFFiciency of HetNets}

This section quantifies the spectral gains of the HetNets for competitive the COE and DCOE configurations in terms of ASE.

\footnotetext{
${ }^{1}$ For the sake of simplicity, the mathematical formula to calculate the adaptive transmit power of the mobile users is not included in this paper. However, such formula can be easily interpreted from [3].
} 
The ASE of the HetNet is defined as the sum of the achievable spectral efficiencies of both the macrocell and the small-cell/D2D communication networks per unit bandwidth per unit coverage area $\left(\mathrm{bps} / \mathrm{Hz} / \mathrm{km}^{2}\right)$, expressed $\mathrm{as}^{2}$ :

$$
A S E=\frac{C_{m}+C_{s}}{\mathrm{~W} \pi\left(R_{m}+R_{s}\right)^{2}}
$$

where $C_{m}$ and $C_{s}$ are the achievable sum-rates of the macrocell and the small-cell/D2D communication networks, respectively, and $\mathrm{W}$ is the total available bandwidth of the network.

Fig. 2 shows the ASE comparison of the cell-edge deployments with increasing macrocell size. The number of small-cells and D2D communication links required to cover the macrocell cell-edge increases with the macrocell radius. It is evident that the DCOE deployment achieves a higher ASE, in comparison with the COE deployments over both the uplink and the downlink directions. This is due to the relatively shorter communication links in the DCOE deployment and the ability of DCOE users to adjust their transmit powers using PC mechanism, resulting in very low power transmissions and reduced interference. The FOE deployment has the highest ASE among the COE deployment strategies due to its smaller cell size, shorter transmitter-receiver distance and the number of supported mobile users connected with each of the femtocells is relatively lower compared to the DOE and ROE scenarios. Consequently, mobile users in the FOE deployment would have more bandwidth available to share between them. Moreover, FOE users are also able to implement PC, which significantly reduces the interference power received at the reference FBS. This directly translates into a higher ASE in the uplink of each femtocell. The DOE deployment, however, has a lower ASE gain in both the uplink and the downlink directions, even though each DA has a relatively higher transmit power compared to the FBS. Each DA supports more mobile users than the FBS does and there is no form of PC mechanism in the uplink, which leads to maximum power transmissions and higher levels of interference. The ROE deployment achieves the least ASE gain of the four deployment strategies due to the larger small-cell coverage of the RBS. Therefore, fewer RBS are needed to cover the cell-edge of

\footnotetext{
${ }^{2}$ A detailed mathematical framework to compute the ASE of HetNets can be found in Section III-A of [3].
} 
the macrocell; this reduces the sources of interference, but not necessarily the interference power, as the relays have a relatively much higher transmit power in the downlink and there is no form of PC mechanism in the uplink. Furthermore, the higher mobile user density associated with the ROE deployment reduces the achievable ASE of this configuration. The DCOE achieves up to $45 \%, 75 \%$, and $90 \%$ more ASE than the FOE, DOE and ROE deployments, respectively, in the downlink; and up to $85 \%, 90 \%$ and $95 \%$ more ASE than the FOE, DOE and ROE deployments respectively, in the uplink. In all four deployment strategies, the ASE starts at a higher level but begins to drop as the radius of the macrocell increases. This is due to the effect of the distance dependent path-loss in the macrocell and more sources of interference at the D2D communication links and reference SBSs as a result of increased deployment.

\section{BACKhaul Power Consumption of HetNets}

This section presents the backhaul power requirements for the cell-edge configurations, the backhaul energy efficiency (BEE) and some backhaul economics.

The end-to-end backhaul power requirement of a HetNet is expressed as:

$$
P_{b h}^{\text {total }}=P_{a g g}+P_{\text {sink }}+\sum_{i=1}^{N} P_{i}
$$

where $P_{a g g}, P_{\text {sink }}$ and $P_{i}$ represent the power consumption of the aggregation node, sink nodes and the backhaul conversion operation either to RF or optical fiber at each of the SBSs, respectively. Note that the backhaul operations of the FOE deployment strictly depend on Internet for backhauling the network traffic to the core network while the DCOE configuration has no need for backhaul, as the D2D communication traffic is not routed via the core network. Moreover, the macrocell BS serves as the sink node for all the SBSs in the DOE and ROE deployments as well as the aggregation node for the sink node and the macrocell BS traffic for backhauling the network traffic to the core network. Furthermore, the sink node acts as the point where the traffic from all the small-cells (excluding FOE deployment) is collected for onward transmission

to the core network. The macrocell BS backhaul link to the core network is assumed to be a high-speed low latency optical fiber cable. 


\section{A. Femtocell Backhauling}

The access network of the femtocell is assumed to be a passive optical network (PON). A single fiber cable from the core network which serves a group of femtocells is fed into an optical line terminal (OLT) which may be located at the local exchange. A passive curb at the local exchange splits the single fiber cable from the OLT into several fibers, each connected to an optical network unit (ONU). Each ONU then serves a single femtocell. The OLTs are connected to edge routers which serve as the femtocell gateways for transmission to the core network. In this paper, we assume the edge router to have a power consumption of approximately $4 \mathrm{~kW}$ and can support up to 40 OLTs. Each ONU and OLT consumes $4.69 \mathrm{~W}$ and $100 \mathrm{~W}$, respectively. The power consumption of the femtocell backhaul network can be computed from [11].

\section{B. DA Backhauling}

Optical fiber cables connect each DA to a sink node switch at the macrocell BS. The sink node switch is connected to an aggregation switch which forward the collected traffic from both tiers to the core network. Each DA has a dedicated interface at the sink node switch and a small-form factor pluggable (SFP) interface within its vicinity, which is used to transmit over the dedicated fiber optic backhaul. The power consumption of the backhaul conversion operation at each SFP is assumed to be $1 \mathrm{~W}$. Furthermore, the power consumption of the sink node is the sum of the power consumption of the sink node switch(es) $P_{\text {switch }}=\left\lceil\frac{N}{\max _{d l}}\right\rceil P_{s}$ and the power consumption for transmitting and receiving the backhaul traffic $P_{\text {sink }}\left(C_{\text {sink }}\right)=N_{u l} P_{u l}$, where $\max _{d l}$ is the maximum number of downlink interfaces at the sink node switch, which we assume to be 24 . This means each sink node switch can support a maximum of 24 DAs. The term $P_{s}$ is the power consumption of the sink node switch with a total traffic of $C_{\text {sink }}$. The number of uplink interfaces is given by $N_{u l}=\left\lceil\frac{C_{\operatorname{sink}}}{U_{\max }}\right\rceil$, where $U_{\max }$ is the maximum transmission rate of an uplink interface. A typical uplink interface can support a maximum transmission rate of 10 Gbps and have a power consumption, $P_{u l}=2 \mathrm{~W}[12]$. 


\section{Relay Backhauling}

Relay nodes usually employ microwave to backhaul their traffic to the macrocell BS. The macrocell traffic is combined with the ROE traffic at the aggregation switch and forwarded to the core network. The power consumption for transmitting and receiving the backhaul traffic over the microwave link at the sink node is given as a function of the total traffic collected from all the relays at the sink node, $C_{\text {sink }}$, such that $P_{\text {sink }}\left(C_{\text {sink }}\right)=37 \mathrm{~W}$ if $C_{\text {sink }} \leq 500 \mathrm{Mbps}$ and 92.5 W, otherwise. The value of $P_{\text {sink }}\left(C_{\text {sink }}\right)$ could be used to compute the power consumption

of the sink node switch(es), with $P_{\text {switch }}=\left\lceil\frac{C_{\text {sink }}}{C_{\text {switch }}^{\text {max }}}\right\rceil P_{s}$, where $C_{\text {switch }}^{\text {max }}$ is the maximum capacity of the switch. The power consumption of the backhaul operation at each relay base station $P_{i}$, is expressed as a function of the $i^{\text {th }}$ relay backhaul traffic $C_{i}$, and is equal to $37 \mathrm{~W}$ if $C_{i} \leq 500$ Mbps and 92.5 W, otherwise [12].

\section{D2D Communication Backhauling}

Unlike the COE configuration where there is a need for backhauling traffic from the small-cells to the core network, the D2D communication network deployment requires no form backhaul as user traffic is not routed through the core network. However, there could be increased control signaling between the macrocell BS and the D2D communication users to measure the link quality, especially during D2D communication initiation. Moreover, the macrocell traffic would still have to be backhauled to the core network, as is the case with the COE configuration.

The summary of backhaul power consumption comparison of the four cell-edge deployment scenarios is shown in Fig. 3. It shows only the backhaul requirement of the small-cells and D2D communication links without considering the backhaul power consumption of the users connected to the macrocell BS. It can be seen that the ROE configuration has the highest backhaul requirement. This is due to the microwave operation at each relay node and the power consumption of the sink node switch. The backhaul power consumption of the DOE scenario increases gradually until there is need for an additional switch at the sink node as the number of DAs deployed to cover the macrocell cell-edge becomes larger than 24, which is the maximum number of downlink interfaces a switch can handle. This results in a sudden leap in the backhaul 
power requirement of the DOE configuration. The power consumption of the FOE configuration is the lowest of the COE deployment technologies and it has a steady increase as the radius of the macrocell increases. The D2D communication configuration, on the other hand, has no backhaul power requirement because D2D communication user data is not routed through the core network, as data is exchanged between communicating D2D users without any intermediary nodes.

\section{E. Backhaul Energy Efficiency (BEE)}

With EE becoming a key design criterion for future networks, it is necessary to examine the backhaul performance of the small-cell networks in terms of EE. In this context, backhaul energy efficiency (BEE) is defined in this paper as the number of bits transmitted per watt of backhaul power consumption in the small-cell network, measured in bits/Joule. Fig. 4 shows the BEE comparison of the small-cell networks, with respect to the macrocell radius. The BEE of the DCOE is not included in this comparison because it has no backhaul power consumption. The FOE deployment has the best BEE in comparison with the other two competitive small-cell deployments. It achieves about $90 \%$ and $280 \%$ more BEE than the DOE and ROE deployments, respectively, at a macrocell radius of $R_{m}=300 \mathrm{~m}$. This is due to the higher $\mathrm{SE}$ of the FOE deployment and its lower backhaul power consumption. However, as the radius of the macrocell increases, the BEE of the FOE begins to fall due to the increase in the number of the FBS resulting in a slight increase in backhaul power consumption. At a macrocell radius of $R_{m}=800 \mathrm{~m}$, the BEE of the FOE deployment is about $15 \%$ and $82 \%$ more than the DOE and ROE deployments, respectively. The DOE deployment achieves between $60 \%$ and 100\% more BEE than the ROE deployment, depending on the macrocell radius. The ROE deployment has the least BEE due to its relatively much lower SE and the higher backhaul power consumption of the deployment.

\section{F. Backhaul Economics}

Higher capacity, perfect synchronization between the BSs and zero latency over an IP network are a must for an ideal cost effective LTE backhaul. Moreover, backhaul with finite latency and 
limited capacity may degrade system performance [13]. Since the LTE networks will be used mostly to carry data rather than voice, one main consideration the operators are faced with is how to migrate toward IP based backhaul. The availability and deployment cost of backhaul are the most important factors in selecting the appropriate cell-edge technology.

Although the cost of a single femtocell is low, the number of femtocells needed to cover the entire cell-edge of the macrocell could be significantly large which could scale up the total cost of the FOE deployment. Moreover, the availability of Internet to provide backhaul to the large number of femtocells to be deployed could present serious concerns. The deployment of DOE could be daunting as the equipment and installation costs of the DAs could be expensive. In situations where there is no provision for Internet or fiber links and it is not economically feasible to provide one (e.g., remote places and difficult terrains), the ROE deployment becomes the only viable solution. Table II shows the summary of the capital (CAPEX) and operational (OPEX) expenditure comparison of the cell-edge deployment configurations.

Recently, millimeter-wave communication has attracted a great deal of interest from academia, industry, and standardization bodies due to its ability to provide multi-gigabit rates required for backhaul transmission between ultra-massive number of small-cells and mobile access network within a macrocell and the line-of-sight nature which helps to control interference [14]. However, millimeter-wave communications require a large directional gain in order to combat their relatively higher path-loss compared with lower frequency cellular network.

\section{Total Power Consumption of HetNets}

The fundamental factors contributing to the increase in the uplink power consumption of mobile communication networks include an increased number of mobile subscribers which was 4.5 billion in 2012 and is expected to reach 7.6 billion by 2020, and the explosive mobile user data traffic volume which attained 45 million TB/year in 2012 and expected to reach 623 million TB/year by 2020 [15]. The cellular BSs and backhaul power requirements are the most intensive components in terms of downlink energy consumption of mobile communication networks. At present, there are more than 4 million BSs serving mobile users, each consuming an average 
of 25 mega Watt Hour (MWH) per year [15]. Hence, it is imperative to analyze the end-to-end power consumption of small-cell deployment configurations in HetNet. This section presents the total power consumption of several cell-edge deployments in order to nominate one as the greener.

Fig. 5(a) shows the uplink power consumption comparison of the cell-edge deployments. It evident that the FOE deployment has the least uplink power consumption which is due to PC for mobile users in both the macrocell and the femtocells and the smaller cell radius of the femtocells. Although the DCOE deployment also employs PC, the path-loss model it employs results in a slightly higher uplink power consumption compared to the FOE scenario. The DOE and ROE deployments, however, have higher uplink power consumption due to the lack of PC mechanism. Furthermore, since the size of the ROE cell is relatively bigger, there are more mobile users in the ROE deployment transmitting with maximum power, compared to the DOE deployment. Thus, the ROE has much higher power consumption in the uplink. The FOE deployment is able to achieve up to $35 \%, 90 \%$ and $95 \%$ reduction in uplink power consumption compared to the DCOE, DOE and ROE deployment scenarios, respectively.

The downlink power consumption, given as the combination of the backhaul power consumption and the power consumed by the macrocell BS and each SBS in the HetNet, is shown in Fig. 5(b). It can be observed that the DCOE deployment has a much lower downlink power consumption compared to the other cell-edge deployment scenarios because only the macrocell BS power consumption and the macrocell backhaul power consumption are considered, as there is no backhaul power requirement in the D2D communication. It is evident that the ROE deployment has the highest downlink power consumption of all the strategies and this is due to the high individual power consumption of relays, which increases as the size of the macrocell increases. The DCOE deployment achieves up to 50\%, 60\% and 85\% downlink power consumption reduction, compared to the FOE, DOE and ROE deployment scenarios, respectively.

The total power consumption of the HetNet, defined as the sum of the downlink and uplink power consumption of the network, is shown in Fig. 5(c). The DCOE deployment exhibits the least total power consumption, and this is due to its low downlink power consumption. This is 
followed by the FOE deployment, which has the least total power consumption of the three COE deployment strategies. This is because of the low power consuming FBS, the lower backhaul power requirement and PC in the uplink of both the macrocell and femtocell networks. The DOE deployment has higher total power consumption because of the higher power consumption of the DAs and the lack of PC in the uplink of mobile users associated with the DAs. The ROE deployment, however, presents the highest total power consumption due to the higher backhaul power requirement and power consumption of the RBSs as well as the lack of a PC scheme in the uplink of the mobile users connected to the RBSs in ROE deployment.

\section{CONCLUSION}

In this paper, a heterogeneous cell-edge deployment of femtocells, DAs, relays and D2D communication was analyzed, with the scope of improving the cell-edge performance of the network. The performances of the cell-edge technologies were compared in terms of their ASE and respective power consumptions, including backhaul, access and aggregation network power consumptions. Simulation results illustrate that the DCOE deployment outperforms all the COE deployment scenarios both in ASE and total power consumption of the network, establishing a more economical and energy efficient solution. However, the DCOE deployment can only be used for close range communication, where the desired mobile users and content are close enough to take part in the D2D communication. This is particularly useful for content distribution and mobile multi-player gaming as well as in mobile relaying. In cases where the possibility of having the desired user and content is very low, FOE could be deployed to support cell-edge users. The FOE deployment has a higher ASE and is a more energy efficient approach than the DOE deployment where fiber is needed, and ROE which requires an additional link and added infrastructure cost to backhaul the traffic to the macrocell BS. Therefore, the use of DAs and relaying to facilitate the edge mobile users is exceptionally expensive under such scenarios, compared to the FOE configuration where the cost of dedicated expensive backhaul is completely eliminated. The ROE deployment provides the least ASE and exhibits the highest total power consumption. 


\section{REFERENCES}

[1] A. Damnjanovic, J. Montojo, W. Yongbin, J. Tingfang, L. Tao, M. Vajapeyam, Y. Taesang, S. Osok, and D. Malladi, "A survey on 3GPP heterogeneous networks," in IEEE Mag. Wireless Communs., vol. 18, no. 3, pp. 10-21, Jun. 2011.

[2] S. Hakola, T. Chen, J. Lehtomäki, and T. Koskela, "Device-to-device (D2D) communication in cellular network - performance analysis of optimum and practical communication mode selection," in Proc. IEEE Wireless Communs. and Networking Conf. (WCNC'2010), Sydney, Australia, Apr. 2010, pp. 1-6.

[3] H. Tabassum, M. Z. Shakir, and M. Alouini, "Area green efficiency (AGE) of two tier heterogeneous cellular networks," in Proc. IEEE Conf. Global Communs., (GLOBECOM'2012), Anaheim, CA, USA, Dec. 2012, pp. 529- 534.

[4] J. Andrews, H. Claussen, M. Dohler, S. Rangan, and M. Reed, "Femtocells: Past, present, and future," in IEEE Journal on Selected Areas Communs., vol. 30, no. 3, pp. 497-508, Apr. 2012.

[5] V. Chandrasekhar, J. G. Andrews, and A. Gatherer, "Femtocell networks: A survey," in IEEE Mag. Communs., vol. 46, no. 9, pp. 59-67, Sep. 2008.

[6] X. You, D. Wang, P. Zhu, and B. Sheng, "Cell edge performance of cellular mobile systems," in IEEE Journal Selected Areas Communs., vol. 29, no. 6, pp. 1139-1150, Jun. 2011.

[7] C. Xiaoming, Z. Zhang, and H.-H. Chen, “On distributed antenna systems with limited feedback precoding: Opportunities and challenges," in IEEE Mag. Communs., vol. 17, no. 2, pp. 80-88, Apr. 2010.

[8] C. Hoymann, W. Chen, J. Montojo, A. Golitschek, C. Koutsimanis, and S. Xiaodong, "Relaying operation in 3GPP LTE: challenges and solutions," in IEEE Mag. Communs., vol. 50, no. 2, pp. 156-162, Feb. 2012.

[9] R. Pabst et. al., "Relay-based deployment concepts for wireless and mobile broadband radio," in IEEE Mag. Communs., vol. 42, no. 9, pp. 80-89, Sep. 2004.

[10] S. Mumtaz, H. Lundqvist, K. M. S. Huq, J. Rodriguez, and A. Radwan, "Smart direct-LTE communication: An energy saving perspective," in Elsevier Journal Ad Hoc Networks, 
vol. 13, no. B, pp. 296-311, Feb. 2014.

[11] J. Baliga, K. Hinton, and R. S. Tucker, "Energy consumption of the internet," in Proc. Joint International Conf. Optical Internet, 2007 and the 32nd Australian Conf. Optical Fibre Technology (COIN-ACOFT'07), Melbourne, Australia, Jun. 2007, pp. 1-3.

[12] P. Monti, S. Tombaz, L. Wosinska, and J. Zander, "Mobile backhaul in heterogeneous network deployments: Technology options and power consumption," in Proc. IEEE 14th. Intl. Conf. Transparent Optical Networks, (ICTON'12), Conventry, UK, Jul. 2012, pp. 1-7.

[13] A. Sanderovich, O. Somekh, H. V. Poor, and S. Shamai, "Uplink macro diversity of limited backhaul cellular network," in IEEE Trans. Information Theory, vol. 55, no. 8, pp. 34573478, Aug. 2009.

[14] T. S. Rappaport et. al., "Millimeter wave mobile communications for 5G cellular: It will work!” in IEEE Journal Access, vol. 1, no. 1, pp. 335-349, 2013.

[15] Energy Aware Radio and Network Technologies (EARTH), http://www.ict-earth.eu, 2012, Online; accessed Mar. 1, 2013. 


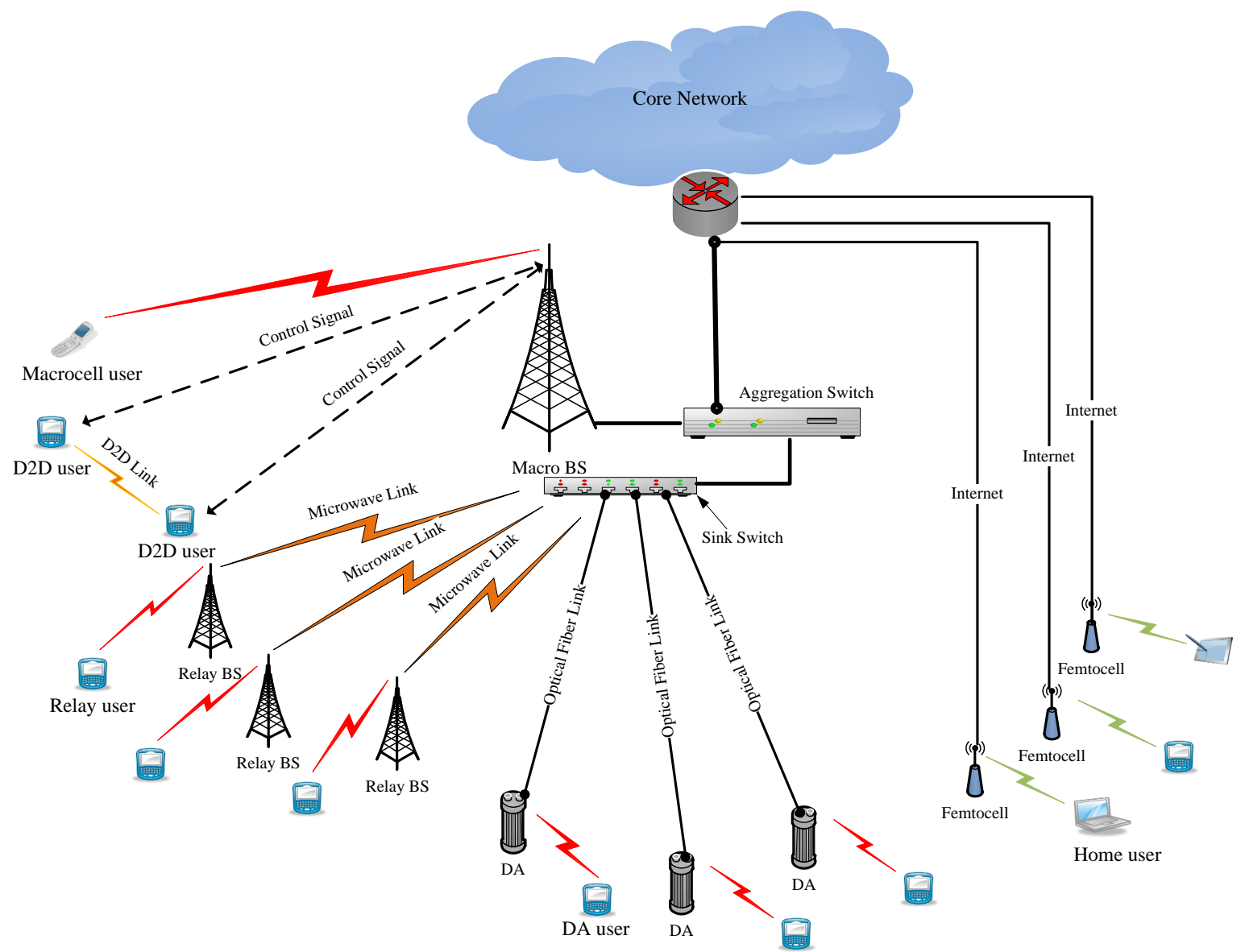

Figure 1. Graphical illustration of the heterogeneous cell-edge deployment where the macrocell infrastructure is supported by cell-on-edge technologies such as femtocells, relays and distributed antennas using dedicated backhaul network, and D2D communication. 


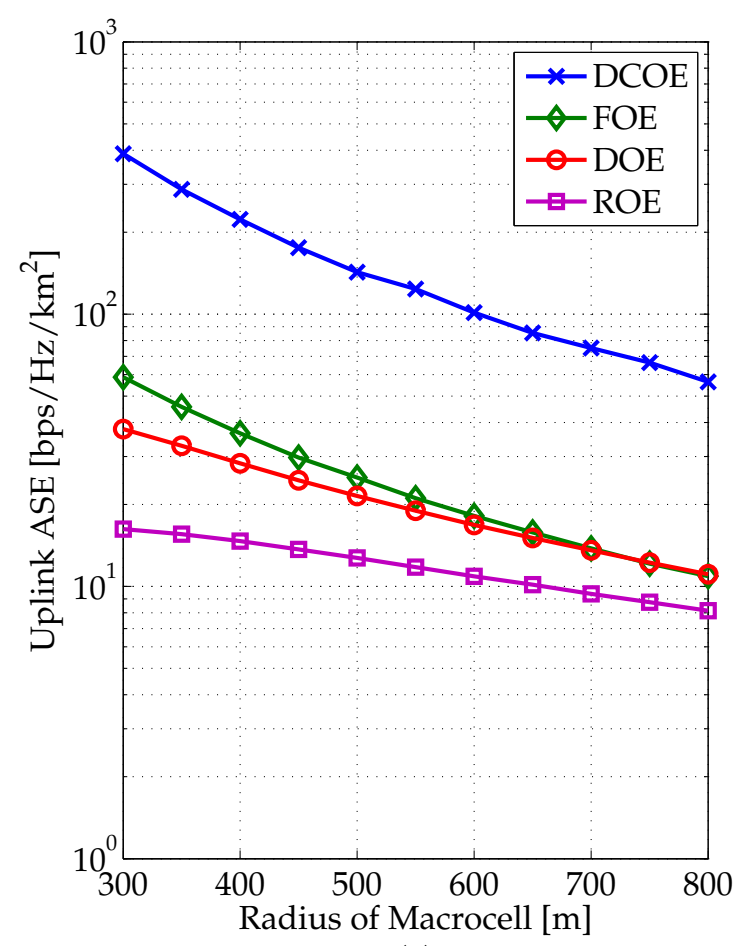

(a)

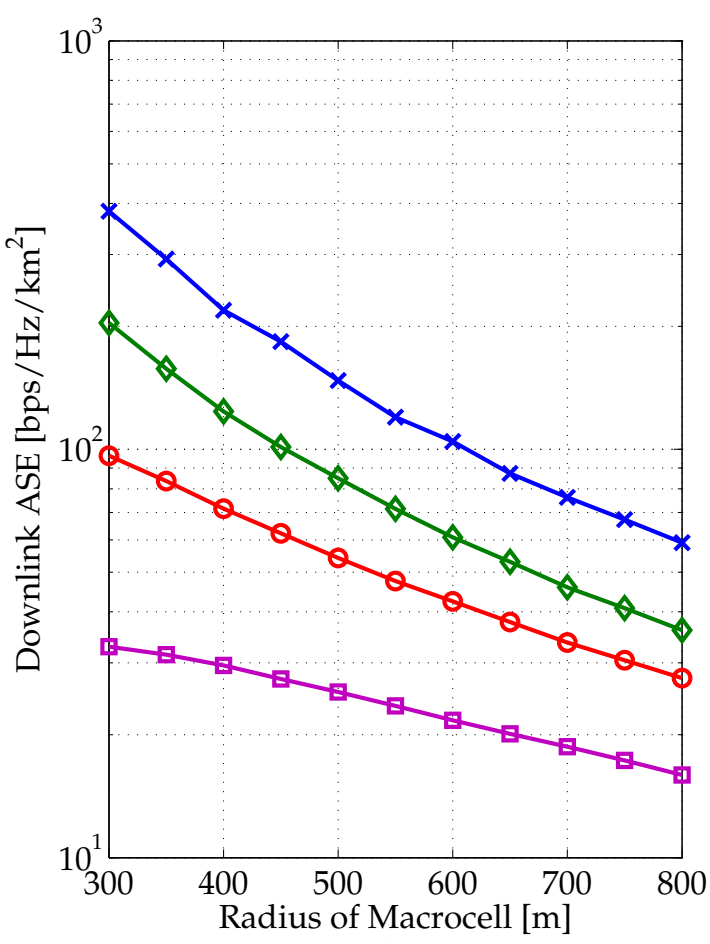

(b)

Figure 2. Summary of area spectral efficiency (ASE) of femto-on-edge (FOE), DA-on-edge (DOE), relay-on-edge (ROE) and D2D-communication-on-edge (DCOE) deployment strategies for (a) uplink and (b) downlink. 


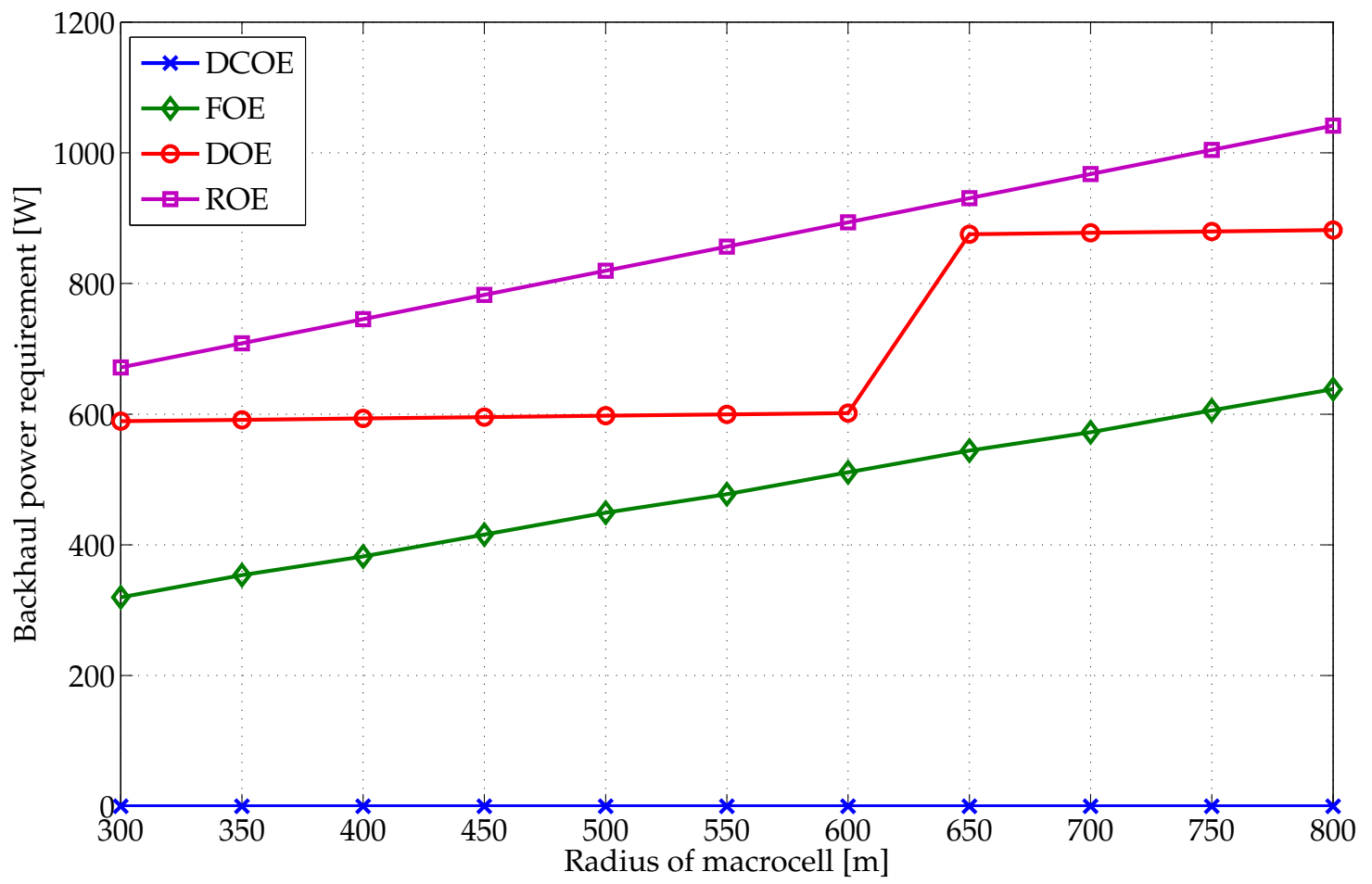

Figure 3. Summary of backhaul power requirement of femto-on-edge (FOE), DA-on-edge (DOE), relay-on-edge (ROE) and D2D-communication-on-edge (DCOE) deployment strategies. 


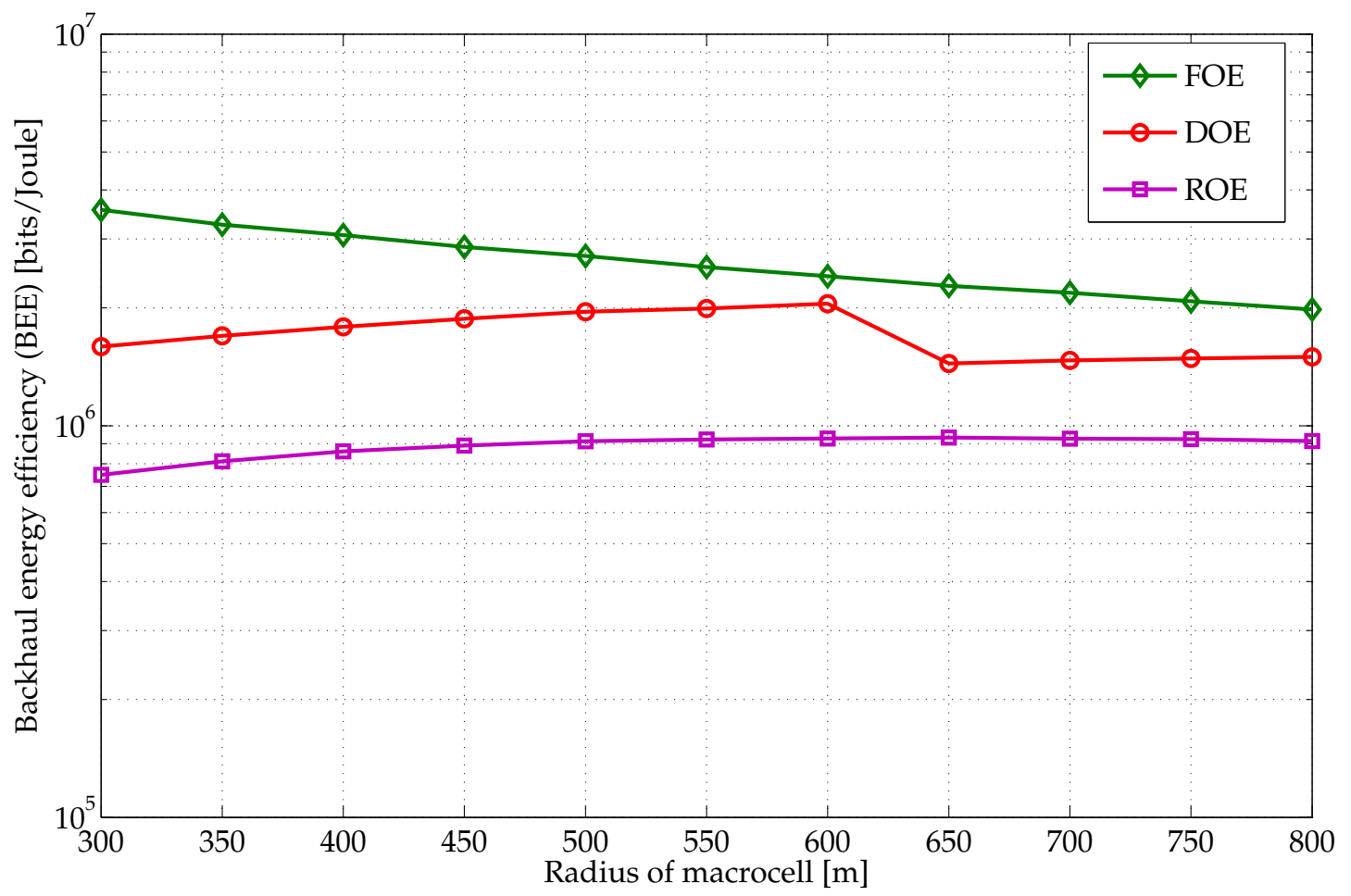

Figure 4. Summary of backhaul energy efficiency (BEE) of femto-on-edge (FOE), DA-on-edge (DOE) and relay-on-edge (ROE) deployment strategies. 


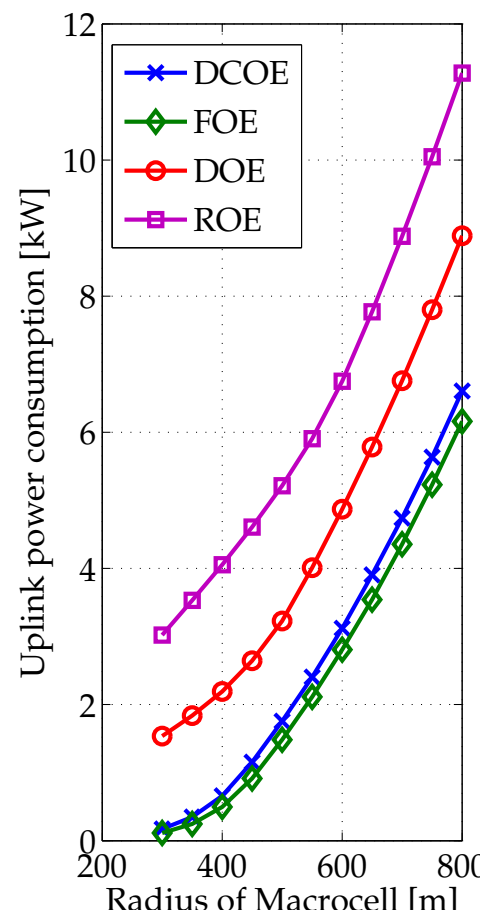

(a)

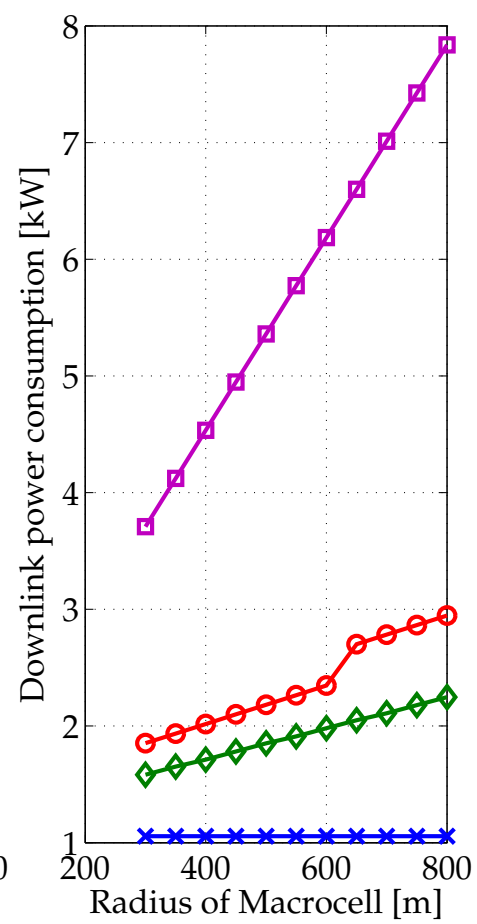

(b)

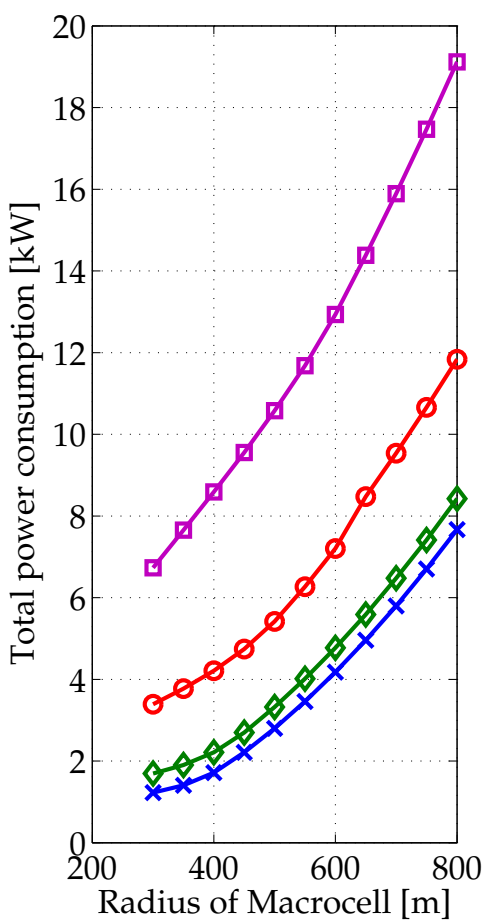

(c)

Figure 5. Summary of power consumptions of femto-on-edge (FOE), DA-on-edge (DOE), relay-on-edge (ROE) and D2Dcommunication-on-edge (DCOE) deployment strategies for (a) uplink; (b) downlink and (c) total power consumption. 
Table I

SIMULATION PARAMETERS

\begin{tabular}{c|c|c|c|c|c|c}
\hline Simulation parameter & Femtocell & DAS & Relay & $\begin{array}{c}\text { D2D } \\
\text { comm. }\end{array}$ & Macrocell & $\begin{array}{c}\text { ggregation } \\
\text { Switch }\end{array}$ \\
\hline Cell radius / range (m) & 30 & 100 & 200 & $20(\max )$ & $300-800$ & - \\
\hline Number of mobile users/cell & 14 & 157 & 629 & - & - & - \\
\hline Downlink tx power (W) & 0.05 & 0.2 & 1 & 0.8 & 20 & - \\
\hline Max uplink tx power (W) & 0.8 & 0.8 & 0.8 & 0.8 & 0.8 & - \\
\hline BS power consumption (W) & 5 & 40 & 376 & - & 783 & 2 \\
\hline$P_{u l}(\mathrm{~W})$ & - & 2 & - & - & - & 1 \\
\hline$P_{d l}(\mathrm{~W})$ & - & 1 & - & - & - & 10 \\
\hline$U_{\max }(\mathrm{Gbps})$ & - & 10 & - & - & - & 24 \\
\hline $\max (d l$ & - & 24 & - & - & - & 0.9 \\
\hline$\alpha$ & - & 0.9 & - & - & - & 24 \\
\hline$C_{s w i t c h}^{\max }(\mathrm{Gbps})$ & - & 24 & 36 & - & - & 300 \\
\hline$P_{\max }(\mathrm{W})$ & - & 300 & 53 & - & - & - \\
\hline$P_{s}(\mathrm{~W})$ & - & 53 & 53 & - & - & - \\
\hline
\end{tabular}

Table II

Cell-edge Deployment EXPENDiture

\begin{tabular}{c|c|c|c|c}
\hline Expenditure & FOE & DOE & ROE & DCOE \\
\hline & CAPEX & $\begin{array}{c}\text { Femtocell } \\
\text { equipment }\end{array}$ & $\begin{array}{c}\text { DA equipment, sink and } \\
\text { aggregation switches, } \\
\text { and fiber backhaul } \\
\text { installation }\end{array}$ & $\begin{array}{c}\text { Macrocll BS } \\
\text { Installation of new } \\
\text { radio masts, backhaul } \\
\text { installation, microwave } \\
\text { antennas, sink and } \\
\text { aggregation switches } \\
\text { No need for } \\
\text { additional cellular } \\
\text { infrastructure and } \\
\text { it is quicker and } \\
\text { easier to implement. }\end{array}$ \\
\hline OPEX & $\begin{array}{c}\text { IP based } \\
\text { backhaul } \\
\text { (SIPTO) }\end{array}$ & $\begin{array}{c}\text { Maintenance of DA } \\
\text { equipment and fiber } \\
\text { backhaul links }\end{array}$ & $\begin{array}{c}\text { Site lease, electricity, and } \\
\text { maintenance of relay and } \\
\text { wireless backhaul links }\end{array}$ & $\begin{array}{c}\text { Signalling over IP } \\
\text { (SoIP). } \\
\text { No maintenance. }\end{array}$ \\
\hline
\end{tabular}

